\title{
Stickler's syndrome: a study of 12 families
}

\author{
A SPALLONE \\ From the Department of Ophthalmology, St Gerardo Hospital, Monza, Milan, Italy
}

SUMMARY Stickler's syndrome is a congenital disease of connective tissue with considerable ocular and non-ocular lesions. This study reports 12 pedigrees ( 10 families and two isolated cases) and evaluates some peculiar ocular aspects not previously reported in the syndrome.

According to recent reports ${ }^{2}$ the term Stickler's syndrome indicates a hereditary disease of connective tissue together with ocular lesions (such as vitreoretinal degeneration and retinal detachment with giant tears), orthopaedic and otolaryngological lesions, a peculiar facial physiognomy, and a characteristic body habitus. During the last year I have studied 12 pedigrees (10 families and two isolated cases), a total of 39 individuals in all, affected by Stickler's syndrome. All 12 propositi had retinal detachment. A diagnosis of Stickler's syndrome was made on the basis of ocular lesions (mainly retinal detachment in high congenital myopia, vitreoretinal degeneration) and non-ocular lesions. Subsequently the family history of the probands was studied to identify affected relatives.

The purpose of my study was to evaluate mainly (1) the association between the ocular and non-ocular pictures in Stickler's syndrome, (2) the variability of expressivity among the different pedigrees and even in the same family among the different generations, and (3) the possibility of a classification into different groups. In addition the study presents some peculiar aspects of the ocular pictures in the patients examined such as ectopia lentis and star shaped or semilunar cataracts, that are probably associated with the general disorders of connective tissue. To my knowledge these aspects have not been reported previously in the description of Stickler's syndrome.

\section{Material and methods}

Of the 39 people examined 20 were males and 19 females. Their ages ranged from 4 to 60 ; however, only two cases were over 40 . A medical history was taken from the 39 patients. The ophthalmic examination placed particular emphasis on high congenital myopia, congenital cataract, ectopia lentis, study of

Correspondence to Dr A Spallone. the angle, examination of the vitreous and the retina by slit-lamp and Goldmann three-mirror lens, observation by binocular indirect ophthalmoscope, fluorescein angiography and sometimes electroretinography, and A and B scan ultrasonography. The non-ocular examination including observation of the characteristic physiognomy, consisting of an Asiatic appearance, flattened nose, long philtrum, small upper lip, receding chin, cleft palate, abnormal teeth, hyperextensibility of the large joints, and a characteristic habitus. $X$-rays of various parts of the body and audiograms were made of all 12 propositi and of some of the other patients.

\section{Results}

OCULAR EXAMINATION Myopia. 87\% of the patients were myopic. In the probands the myopia was always over 10D sph and increased with age, contrary to what has been previously reported. Lacquer cracks or neovascular membranes were not found in any of the myopic patients.

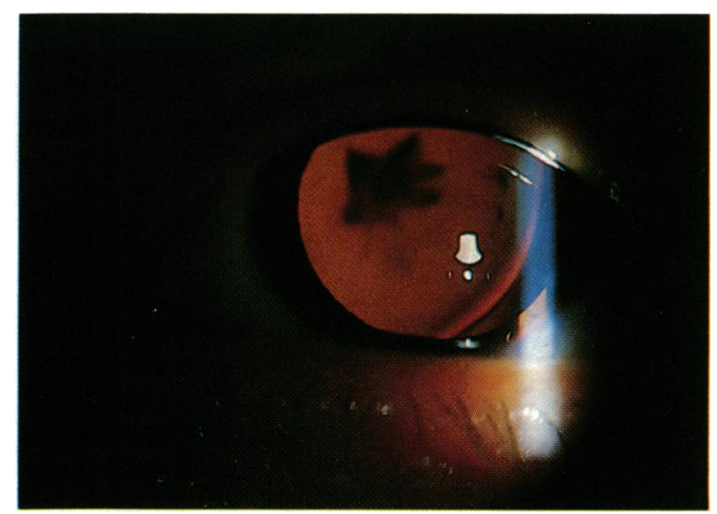

Fig. 1 Star shaped cataract with ectopia lentis. 


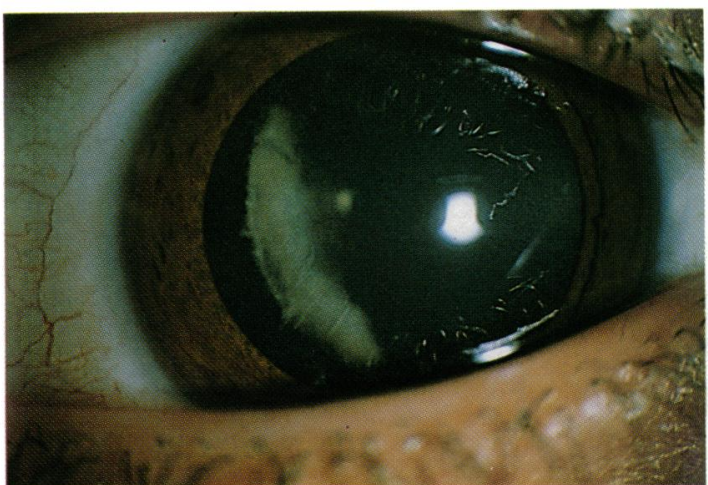

Fig. 2 Congenital cortical cataract with semilunar aspect.

Lens. $49 \%$ of the patients had presenile cataract. Some of these were subcapsular cataracts, while others were cortical opacities with peculiar features (star shaped or semilunar) (Figs. 1,2).

Ectopia lentis. This was present in five cases $(12.8 \%)$; in two it was bilateral and congenital (Fig. 3). In these two last cases the lenses were dislocated upwards and temporally, while in the remaining three cases the lenses were dislocated into the vitreous. Owing to the youth of the patients it was difficult to evaluate the possibility of previous trauma. It was possible to exclude other ocular and systemic disorders that generally cause ectopia lentis. ${ }^{+}$

Intraocular pressure. Ocular hypertension was present in $10 \%$ of the patients. Few cases had glaucomatous field loss.

Angle aspect. In $26 \%$ of the patients long and

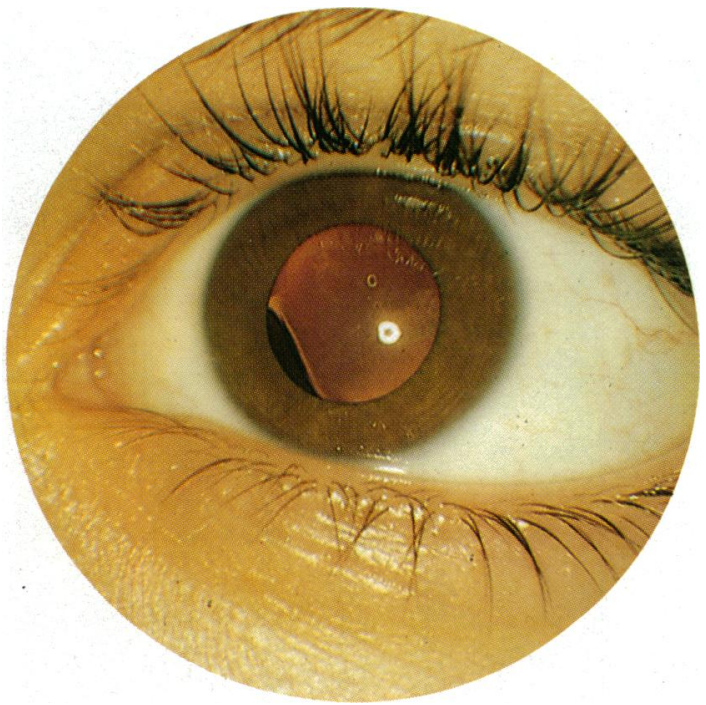

Fig. 3 Ectopialentis.

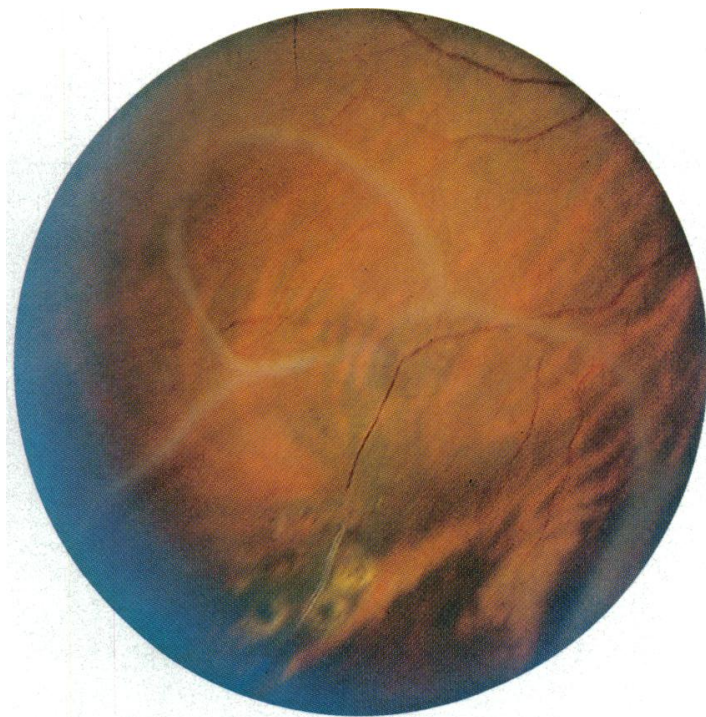

Fig. 4 Large syneresis with pocket and circumlinear equatorial adhesions.

thick iris processes stretched to the trabecular meshwork and covered part of the ciliary face and scleral spur in the anterior chamber angles. This picture is very similar to that seen in the Marfan syndrome, ${ }^{56}$ Marfan-like syndrome, ${ }^{7}$ Stickler's syndrome, ${ }^{8}$ and sometimes pathological myopia.

Vitreous. $85 \%$ of the patients and all probands had vitreous abnormalities. The vitreous often appeared to be optically empty on slit-lamp examination. A syneresis with fenestrated vitreous membranes and circumlinear equatorial adhesions was present in other patients (Fig. 4).

Fundus. There were alterations in both posterior and peripheral retina in $87 \%$ of the patients. Notwithstanding the high degree of myopia, a myopic crescent was not often seen. This may have been due to the fact that some of the patients were very young. It has been noted that congenital myopia may increase with age, and so some characteristic degenerations, such as myopic crescent, may develop later. Sometimes an irregular and deformed appearance of the optic disc was seen, associated with straightening of retinal vessels at the posterior pole. Most patients were found to have hypopigmentation of the fundus (fundus flavus). Some patients showed a typical radial perivascular chorioretinal degeneration (Fig. 5).

But the most characteristic appearances were in the peripheral retina and, in particular, in the temporal periphery. These areas were studied by fluorescein angiography and ophthalmoscopy. In many cases it was possible to observe interruption of 


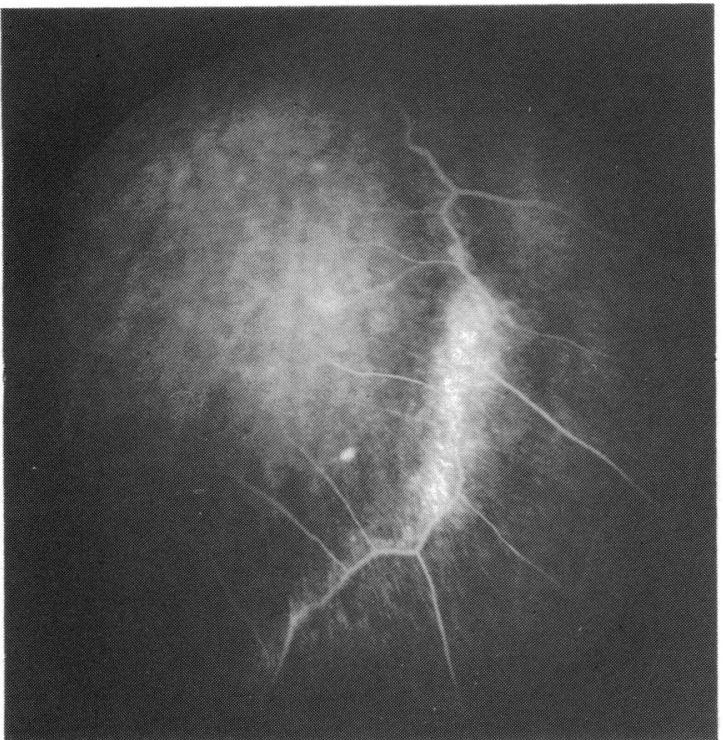

Fig. 5 Radial perivascular chorioretinal degeneration.

the retinal vessels in equatorial areas and sometimes further posteriorly (Figs. 6a and 6b). In some cases the retinal vessels became sheathed and showed signs of leakage (Fig. 7). Hard exudates were present where the leakage was profuse. In other cases nonperfusion was observed, with a notable alteration in the configuration of the retinal vessels (Fig. 8). Although the same type of vascular changes seen in our patients may occur in dominant exudative vitreoretinopathy (DEVR), ${ }^{910}$ in no case did we detect the dense, radially orientated capillaries that are characteristic of DEVR. Twenty-one of the 39 patients $(55 \%)$ had retinal detachment and six cases had giant retinal tears greater than $180^{\circ}$. A female child with a Pierre-Robin anomaly had a $360^{\circ}$ giant retinal tear. In all these cases the other eye showed vascular and retinal abnormalities. Electroretinography gave normal results in 12 cases examined.

\section{NON-OCULAR EXAMINATION Orthopaedic} results. Abnormalities of the joints were present in many patients, including laxity and hyperextensibility $(23 \%)$, width of joints $(21 \%)$, articular click $(44 \%)$, and morning stiffness $(21 \%)$. The patients with hyperextensibility of the joints did not have excessively wide joints, while, on the contrary, patients with reduced flexibility had notably wider joints. $21 \%$ of the patients had a Marfanoid habitus, while $13 \%$ had a Weill-Marchesani habitus. The last two types of habitus were also present in two members of the same family.

$X$-ray results. These have been previously

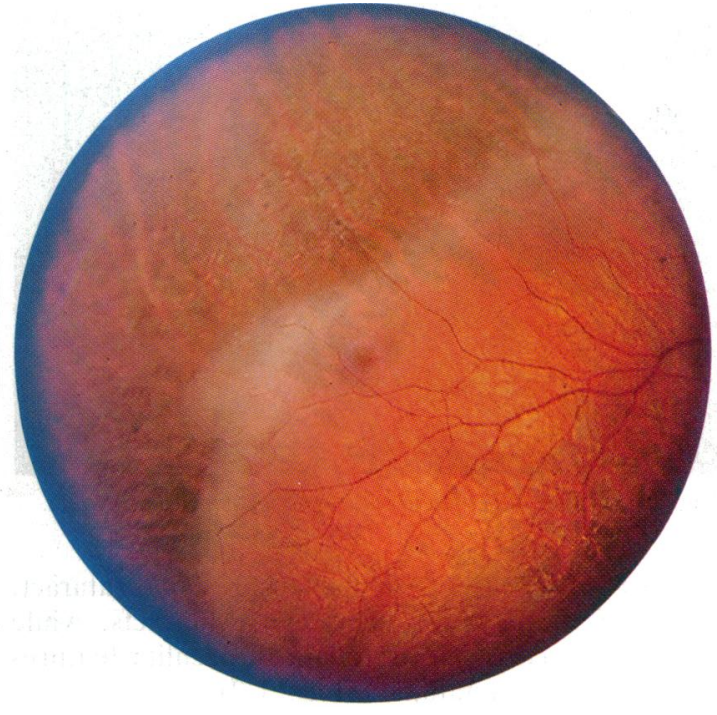

Fig. $6 a$

Figs. 6a, $6 \mathrm{~b}$ Temporal quadrant of the fundus: note the interruption of the retinal vessels and no development of the retinal tissue.

described in detail..$^{2311}$ The roentgenographic studies showed evidence of generalised epiphyseal dysplasias which may affect any of the joints. All 12 propositi and some other patients showed this type of lesion.

Facial appearance. Many of the patients had a peculiar physiognomy characterised by a flattened bridge of the nose $(41 \%)$, maxillary hypoplasia

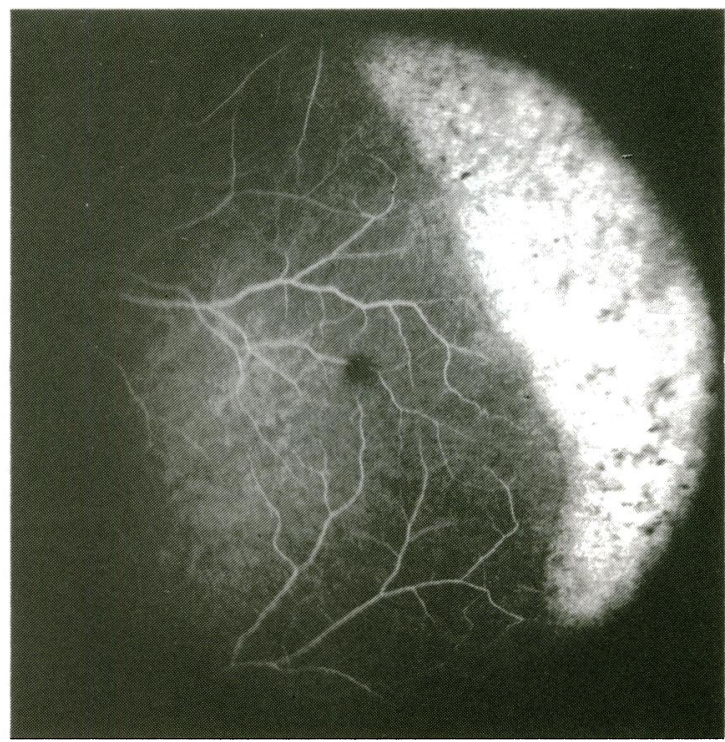

Fig. $6 b$ 


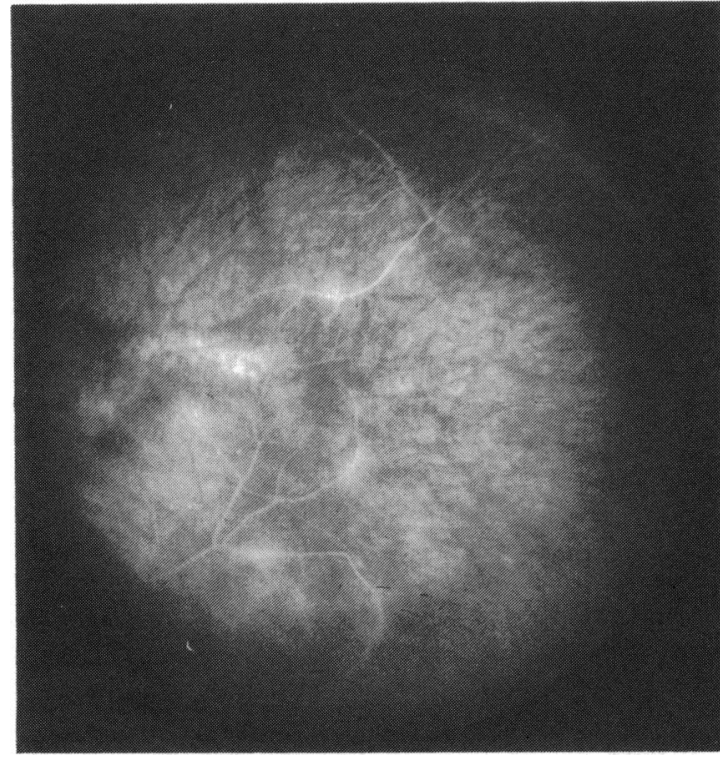

Fig. 7 Mild leakage of the retinal vessels near the posterior pole.

$(31 \%)$, epicanthus $(15 \%)$, receding chin $(18 \%)$, small upper lip $(23 \%)$, long philtrum $(13 \%)$, and trycomegalia (13\%).

Otolaryngology signs. Cleft palate was present in $18 \%$ of the patients, while $31 \%$ had a highly arched or submucous palate. Two of these patients had been diagnosed at birth as having Pierre-Robin syndrome. About $46 \%$ had malocclusion and $21 \%$ hearing loss.

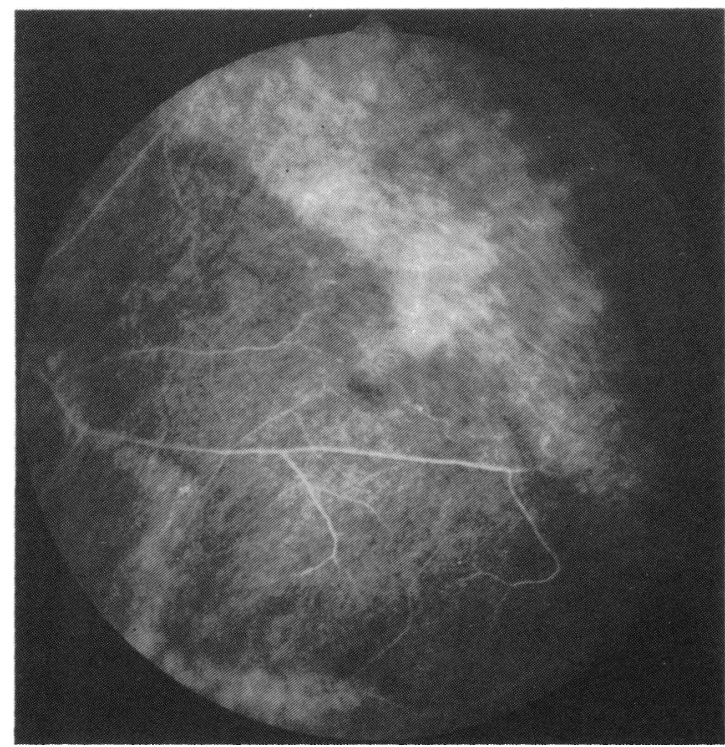

Fig. 8 Arrest of the peripheral vascularisation with alterations in the configuration of the retinal vessels.
These last percentages are lower than those reported by other workers,' probably owing to the youth of my patients.

Genetic results. Two of my patients were sporadic cases and therefore probably new mutations. In the remaining 10 pedigrees an autosomal dominant inheritance was always clearly demonstrated.

\section{Discussion}

Before the classic description of Wagner in 1938 of a large Swiss family with hereditary hyaloideoretinal degeneration $^{12}$ some authors had reported retinal detachment in some pedigrees. For example, Lang in 1885 reported retinal detachment in two brothers. ${ }^{13}$ In 1930 Bane also reported retinal detachment in a family. ${ }^{14}$ These two last works do not describe the vitreous degeneration, and the pedigree of Wagner does not present retinal detachment. In 1966 Jansen reported on two Dutch families and 44 cases which showed hereditary hyaloideoretinal degeneration; in them retinal detachment was quite frequent. ${ }^{\text {is }}$ One year before Stickler $e t a l .{ }^{16}$ had described for the first time the association between vitreoretinal problems and premature degenerative changes in various joints under the name 'hereditary arthro-ophthalmopathy.' Subsequent descriptions ${ }^{3 \times 1117}$ reported large pedigrees in which a characteristic physiognomy, cleft palate, and orthopaedic lesions were associated with vitreoretinal degeneration and retinal detachment. Knobloch" radiologically examined families affected by Wagner's syndrome and discovered that 20 of the 21 patients suffered from arthropathies of varying degrees. These reports have shown that in Stickler's syndrome a pathological hereditary association between ocular and non-ocular disorders may exist and that the lesions are extremely variable in their expressivity. Maumenee ${ }^{1 x}$ has classified these syndromes into six distinct groups and considers that probably two or more gene loci are involved in them.

My studies show that in Italy Stickler's syndrome is rather common. It is important to make an early diagnosis so that prompt treatment of the ocular and non-ocular disorders and genetic counselling may be given. Furthermore, in agreement with other authors ${ }^{x}$ I believe that Stickler's syndrome is a single entity. In fact none of the families examined in this study fitted exactly into any of the recognised categories proposed by Maumenee. For example, when the first 10 patients of one of our families were visited in our department, they were initially diagnosed as having Wagner's syndrome. We then examined two other members of this family who suffered from typical joint changes described in Stickler's syndrome. In another family the father, who is completely blind from bilateral retinal detachment, might be classified 
as having a Weill-Marchesani-like syndrome (according to Maumenee). By contrast in the same family the two children, 14 and 13 years old, with serious retinal problems, show a Marfanoid habitus. Moreover, in the remaining pedigrees the clinical pictures are not always unequivocal, and various signs of connective tissue diseases frequently overlap. From the genetic point of view I think that probably only a single gene is involved. It has an autosomal dominant inheritance with a large range of expressivity and large pleiotrophic effects. The phenotypic consequences probably differ owing to the correlation between this hypothetical gene and the genetic background.

There are many ways in which disorders of connective tissue may affect the ocular system. The main ocular disorders seen in my studies of Stickler's syndrome are (1) high congenital myopia, (2) vitreoretinal and chorioretinal degeneration, and (3) retinal detachment. As other authors ${ }^{315}$ have found, I believe that high myopia in my patients is always congenital. In fact most of the myopic patients were very young. I had frequently observed some peculiar features of pathological myopia such as 'fundus flavus' and retinal vessels of an irregular configuration. This finding is important because pathological myopia might always be considered a heredodegenerative entity, which is entirely different from ocular elongation and is often associated with other heredodegenerative conditions. ${ }^{192}$ In the patients examined, detachment of the retina was the most disabling consequence of this syndrome. It is worth noting that the ophthalmoscopic and fluorangiographic aspects of the fellow eye in all patients with monolateral retinal detachment were very unusual in particular in the temporal mid-periphery, where it was possible to see the interruption of the retinal vascularisation near the equator with atrophic changes of the retina. Knowledge of these changes is important for early prophylaxis of the retinal detachment.

Hagler et al. ${ }^{21}$ reported a group of 33 patients with an unusual but specific type of retinal degeneration characterised by pigment clumping round retinal vessels, with underlying atrophy of the pigment epithelium, This degeneration was frequently associated with malignant forms of retinal detachment. In the majority of patients in Hagler's series a family pattern was present. This author thought the degeneration probably represented a distinct clinical entity different from that described by Wagner. On the contrary, I saw this type of retinal malformation in many of my patients who showed clear signs of Stickler's syndrome. I believe that these alterations are due to disturbances in the complex correlation between the developing retinal vascular system and the vitreous. This interference leads to inhibitions in the normal development of the retinal vessels and therefore to malformations of the inner retinal layers. Apart from high myopia, retinal detachment, and vitreous degeneration, other ocular aspects such as ectopia lentis, which have never been described before in Stickler's syndrome, were found in my patients. As others have noted ${ }^{\times 14}$ some of my patients also showed angle abnormalities, as frequently seen in the Marfan syndrome and sometimes in pathological myopia. In my study this aspect was not associated with a Marfanoid habitus or with ectopia lentis. This result confirms the overlapping between the different signs of the Stickler's syndrome.

My studies have shown that Stickler's syndrome is frequent and often incorrectly diagnosed. It is therefore extremely important to continue to study the biochemical processes of connective tissue disorders in this syndrome.

\section{References}

1 Liberfarb R, Hirose T. The Wagner-Stickler's syndrome. Birth Defects 1982; 18: 525-38.

2 Weingeist IA, Hermsen V, Hanson JW, Bumsted RM, Weinstein SL, Olin WH. Ocular and systemic manifestations of Stickler's syndrome: a preliminary report. Birth Defects 1982; 18: 539-60.

3 Herrmann J, France TD, Spranger JW, Opitz JM. The Stickler's syndrome. Birth Defects 1975; 11: 76-101.

4 Nelson LB. Maumence I. Ectopia lentis. Surv Ophthalmol 1982: 27: 145-60.

5 Burian HM, Allen L. Histologic study of the chamber angle of patients with Marfan's syndrome. Arch Ophthalmol 1961; 65: 323-33.

6 Burian HM, von Noorden GK, Ponseti IV. Chamber angle anomalies in systemic connective tissue disorders. Arch Ophthalmol 1960; 64: 671-80.

7 Cotlier E, Reinglass H. Marfan-like syndrome with lens involvement. Arch Ophthalmol 1975; 93: 93-106.

8 Nielsen CE. Stickler's syndrome. Acta Ophthalmol (KGL) 1981; 59: $286-95$.

9 Criswick VG, Schepens CL. Familial exudative vitreoretinopathy. Am J Ophthalmol 1969; 68: 578-94.

10 Van Nouhuys CE. Dominant exudative vitreoretinopathy and other vascular developmental disorders of the peripheral retina. Monogr Ophthalmol 1982: 5.

11 Knobloch WH. Inherited hyaloideoretinopathy and skeletal dysplasia. Trans Am Opthalmol Soc. 1976; 73: 417-51.

12 Wagner $\mathrm{H}$. Ein Bisher unbekanntes Erbleiden des Auges (degeneratio hialoideoretinalis hereditaria), beobachtet in Kanton Zürich. Klin Monatsbl Augenheilkd 1938; 100: 840-57.

13 Lang W. Detachment of the retina in the yellow spot region: two cases, brother and sister. Ophthalmic Review 1885; 4: 121.

14 Bane WC. Familiar retinal detachment. Am J Ophthalmol 1930; 13: 1004-5.

15 Jansen LMAA. Hei Syndroom van Wagner (degeneratio hialoideoretinalis hereditaria). Nijmegen University thesis. Van Gorcum, 1966.

16 Stickler GB, Belau PG, Farrell FJ, et al. hereditary progressive arthro-ophthalmopathy. Mayo Clin Proc 1965; 40: 433-55.

17 Regenbogen L. Hereditary vitreoretinal degeneration, cleft lip and palate, deafness and skeletal dysplasia. Am J Opthalmol 1980; 89: 414-8. 
18 Maumenee I. Vitreoretinal degeneration as a sign of generalised connective tissue diseases. Am J Opthalmol 1979; 88: 432-49.

19 Black RK. Degenerative myopia. In: Krill AE, ed. Hereditary retinal and choroidal diseases Hagerstown: Harper and Row, 1977: 2.
20 Curtin BS. The pathogenesis of congenital myopia. Arch Ophthalmol 1963; 69: 166-75.

21 Hagler WS, Crosswell HH Jr. Radial perivascular chorioretinal degeneration and retinal detachment. Ophthalmology 1968; 72: 203-16.

Accepted for publication 13 August 1986. 